\title{
Performance of goldfish trained in allocentric and egocentric maze procedures suggests the presence of a cognitive mapping system in fishes
}

\author{
FERNANDO RODRIGUEZ, EMILIO DURAN, \\ JUAN P. VARGAS, BLAS TORRES, and COSME SALAS \\ University of Seville, Seville, Spain
}

\begin{abstract}
Goldfish were trained to obtain food in a four-arm maze placed in a room with relevant spatial cues. Four experimental conditions were run: allocentric, egocentric, egocentric + allocentric, and control. Relative to controls, all groups were able to solve the different tasks with high accuracy after 1 week of training. Subsequent transfer tests revealed place and response strategies for allocentric and egocentric groups, respectively, and both types of strategies for the ego-allocentric group. Moreover, the allocentric group showed the capacity to choose the appropriate trajectory toward the goal, even from novel starting points, presumably by using the distal cues as a whole. The results suggest that, in addition to using egocentric strategies, goldfish are able to solve spatial tasks on the basis of allocentric frames of reference and to build complex spatial cognitive representations of their environment.
\end{abstract}

Fishes travel across a wide range of distances with surprising efficiency, whether in intercontinental migrations or on excursions within their habitual living areas. These travels indicate remarkable spatial abilities of fishes in navigating, orienting themselves, piloting, and recognizing their environment. Research in this field has been focused mainly on the innate fixed patterns of behavior and on sensory, ecological, and zoological factors. What seems to be underestimated in this research, though, is the possibility that spatial behavior is a flexible process that involves learning and memory mechanisms and cognitive phenomena (for a review, see Dodson, 1988). Such mechanisms are indicated by a number of naturalistic and experimental studies, such as the pioneer work of Aronson $(1951,1971)$ that showed that the gobiid fish Bathygobius soporator uses learned information about the spatial relationships of tide pools, or topographical memories acquired during exploration, to orient itself accurately. In fact, complex spatial learning and memory capabilities in fishes can be inferred from recent naturalistic studies (Hallacher, 1984; Helfman, Meyer, \& McFarland, 1982; Helfman \& Schultz, 1984; Markevich, 1988). Furthermore, it has been suggested that some fishes use simul-

We wish to thank J. Bruce Overmier and Catherine Thinus-Blanc for their helpful suggestions and critical reading of a previous version of this manuscript. We also greatly appreciated the comments of Peter J. Urcuioli and two anonymous reviewers, which led to improvements in this paper. This work has been supported by grants from CICYT, the Junta de Andalucía, and the FISSS. Requests for reprints should be addressed to F. Rodríguez, Laboratorio de Psicobiología, Grupo Neurobiología de Vertebrados, Departamento de Fisiología y Biología Animal, Facultad de Psicología, Avda. San Francisco Javier s/n, 41005 Sevilla, Spain. taneously a system of learned, route-specific landmarks in conjunction with solar compass orientation and perhaps a cognitive map (Reese, 1989). In summary, naturalistic fish studies describe in detail the various aspects of fishes' travel but are rather imprecise about the spatial strategies that may be employed in travel.

Spatial learning and memory capabilities of fishes have been examined more closely in laboratory experiments. For example, the performance of Siamese fighting fish (Betta splendens) in the eight-arm maze procedure shows a strong algorithmic component in the determination of the sequential choices, although some small amount of spatial memory is involved in the task (Roitblat, Tham, \& Golub, 1982). Goldfish (Carassius auratus) have been shown to remember the spatial position of food patches in a tank (Pitcher \& Magurran, 1983), to use landmarks as indirect spatial reference points (Warburton, 1990), and also to swim in a constant direction relative to visual cues, even if they approach the goal from opposite directions. This ability could reflect a capacity for discriminating spatial relationships in the environment independently of a body-centered reference system (Ingle \& Sahagian, 1973). Fishes also show an organized pattern of exploration when they are introduced into a novel environment, indicating some degree of spatial knowledge and memory (Kleerekoper, Matis, Gensler, \& Maynard, 1974), and they can detect environmental modifications, reacting with increased exploratory activity (Welker \& Welker, 1958). Recently, Teyke (1989) reported that following release in unfamiliar surroundings, or after alteration of a familiar environment, the blind cave fish (Anoptichthys jordani) swim around boundary features and increase their swimming velocity, possibly to optimize lateral line organ 
stimulation. He proposed that fishes develop an internal map of their environment that allows them to swim at velocities below the optimal range in familiar environments without observable deficits in avoiding obstacles.

The preceding studies suggest the presence of complex spatial learning and memory capabilities in fishes and the participation of cognitive mechanisms in the solution of spatial problems. The concept of a cognitive map, first suggested by Tolman (1948), has become a major theoretical construct since the formulation of the spatial learning and memory theory of O 'Keefe and Nadel (1978). This theory postulates the presence of two different spatial behavior systems: the cognitive mapping and taxon systems. They appear to act independently, but, under the appropriate conditions, they may cooperate or conflict with each other (O'Keefe \& Nadel, 1978; Schenk \& Morris, 1985; Whishaw, 1989; Whishaw \& Mittleman, 1986). The cognitive mapping system is based on allocentric strategies, it involves an internal map-like representation of the objective spatial relationships among different landmarks, and it is independent of any particular view of the surrounding (Mazmanian \& Roberts, 1983; Morris, 1981; O’Keefe \& Conway, 1978; Suzuki, Augerinos, \& Black, 1980). The internal spatial representations of this system provide animals with greater flexibility in their spatial behavior than that offered by taxon strategies. Hence, animals that rely on such a cognitive, topographical representation can locate a place from different directions and adopt novel routes from points previously unvisited, even in the absence of any specific cue associated with the goal. By contrast, the taxon systems are based on purely egocentric strategies and include guidance and orientation. The guidance strategies involve approaching or avoiding particular cues, whereas orientation indicates a body-centered turn in response to a cue. Properties of these two systems have been described elsewhere (O`Keefe \& Nadel, 1978) and have been thoroughly studied in mammals (Nadel, 1991; O'Keefe \& Nadel, 1978) and birds (Bingman, 1990; Bingman, Bagnoli, Ioalé, \& Casini, 1989). To date, however, the relevance of such systems is not well known in fishes. Comparative research with fish could reveal interesting insights about the evolutionary and adaptive importance of these strategies in vertebrates.

The general aim of this work was to determine whether fishes are able to solve spatial tasks by using allocentric frames of reference in addition to the purely body-centered ones. Furthermore, we investigated whether fishes could build and use internal maps of their environment, and whether such hypothetical internal maps show some of the properties described by O`Keefe and Nadel (1978). With this purpose, the performances of goldfish trained in allocentric versus egocentric tasks in a four-arm maze were compared. In particular, the following questions were addressed: (1) Are differences in learning evident in allocentric versus egocentric tasks? (2) What kind of cues is used to solve allocentric tasks? For example, do goldfish use distal visual cues to locate a place? (3) Can allocentric and egocentric strategies act in concert or are they mutually exclusive? (4) Does prolonged training differentially affect these strategies, and might they be revealed by the reversal of the task?

\section{METHOD}

\section{Subjects}

Goldfish (Carassius auratus) obtained from a local supplier were maintained, for 2 months prior to the experiments, in small groups in glass aquaria with aerated filtered water at $20^{\circ} \mathrm{C} \pm 2^{\circ}$. They were kept on a 14:10-h light:dark cycle and given food ad libitum during this time.

Thirty-two animals, $10-14 \mathrm{~cm}$ in length, were randomly selected from stock tanks and assigned to four groups. They were housed in similar aquaria and deprived of any food for 2 days before the experiment. The animals were recognized by phenotypical characters. Throughout the experiment, the fishes received only the five food sticks they obtained every day in the experimental session. Each stick was $20 \pm 0.8 \mathrm{mg}$ (mean $\pm S D$ ) of dry food for pond fish (Tetra pond).

\section{Apparatus}

The apparatus was an elevated four-arm maze made out of transparent glass; the floor was covered with opaque white Perspex. Only three of the arms were used on any one conditioning trial. Each arm was $80 \mathrm{~cm}$ long, $17 \mathrm{~cm}$ wide, and $20 \mathrm{~cm}$ high, with a 17 $\mathrm{cm}$-square central platform. For each trial, the startbox was delimited by a $20-\mathrm{cm}$-high opaque Perspex guillotine door placed $16 \mathrm{~cm}$ from the end of the arm. The door was controlled by a hand operated device. Before every session, the maze was filled with water at $20^{\circ} \mathrm{C} \pm 1^{\circ}$ to a depth of $15 \mathrm{~cm}$ and aerated at the end of each arm. At the end of each of the two non-start arms, a food holder was attached by suction to the floor of the arm. The food holder was composed of a $90^{\circ}$ curved glass bar $(5 \mathrm{~mm}$ in diameter). The upper segment of the bar was horizontal, elevated $5 \mathrm{~cm}$ above the maze floor. At one end of the bar was inserted a dark latex tube (1.5 cm long), which could enclose one stick of food. This food was not visible, and the fish took it out by suction. The

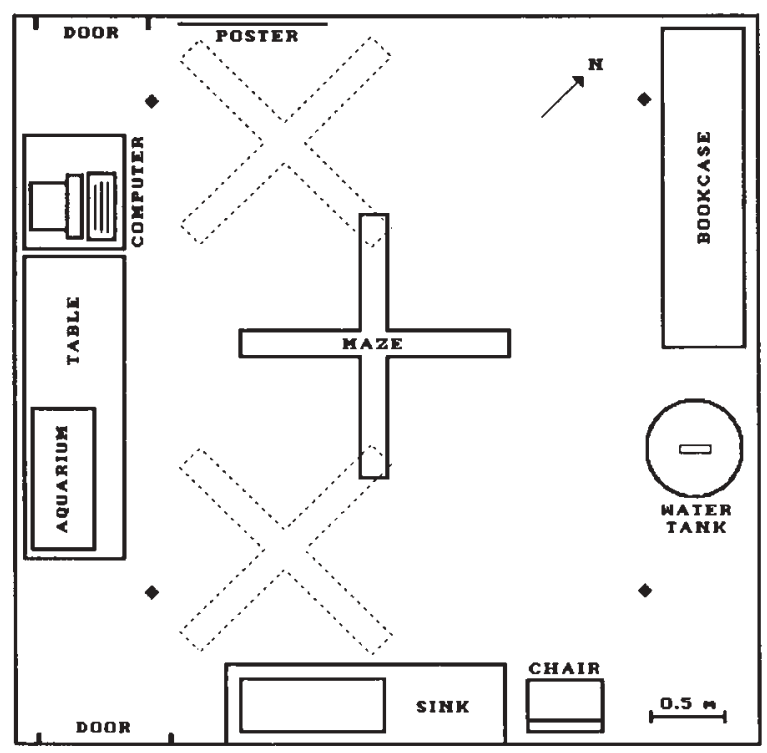

Figure 1. Plan to scale of the experimental room and maze. The maze location is shown in its normal position (solid line) and in its displaced positions for Type 2 transfer tests (dashed lines). Rhombuses indicate the anchorage points for the curtains. 
goal arm was the one containing the baited food holder. The maze was placed in the center of a room $4.50 \times 4.64 \times 2.60 \mathrm{~m}$, which was illuminated by four overhead lights. The plan of the room, the various extramaze cues, and the location of the maze during training are shown in Figure 1.

The experimenter, located in an adjoining room, controlled the door of the maze and, through a small opening, observed the behavior of the animal. When called for, the maze could be surrounded by thick brown curtains, which hung from ceiling to floor, and which formed a 3-m-square enclosure. These curtains excluded virtually all extramaze visual cues. The maze was randomly rotated between experimental sessions in order to preclude the use of any possible intramaze cues.

\section{Procedure}

Pretraining. To get the fish to obtain food from the food holder, they were placed for 2 consecutive days in a little aquarium provided with a food holder, which was rebaited until each fish ate five times. This aquarium was located outside of the experimental room. On the same days, two 2-h sessions were also run in which the subject was placed in the experimental room and allowed to explore freely throughout the maze. During these sessions, the food holders were removed from the apparatus. Following this exploration period, the animals were deprived of any food for 2 days prior to the experiment proper.

Training. Animals were assigned to four different groups $(n=8)$ for training in one of the following procedures: allocentric, egocentric, ego-allocentric, and control (see Figure 2).
Allocentric procedure. In this group, two opposite start points were randomly used $(50 \%$ each), and the fish were always rewarded at the extreme of the goal arm situated in the same place of the room (Figure 2A). This procedure was used to determine whether or not the fish could learn to go to a rewarded site solely on the basis of extramaze cues. No fixed-turn direction was relevant to task solution, because, depending on the location of the startbox, subjects were required to make a left- or right-hand turn.

Egocentric procedure. For this group, two opposite start arms were randomly used ( $50 \%$ each), and for all trials, the goal arm was determined by a fixed-turn response (e.g., always left) relative to the start arm (Figure 2B). This procedure determined whether or not the fish could choose the correct arm on the basis of a specific turn response with extramazes cues irrelevant to task solution.

Ego-allocentric procedure. The fish in this group left a startbox that was always situated in the same place of the room, and they were rewarded exclusively in a goal arm located in another constant place (Figure 2C). This task thus allowed selection of the correct arm on the basis of a specific turn direction and/or extramaze cues.

Control procedure. The fish in the control group left from a startbox that was always situated in a fixed place of the room. However, their training involved two possible correct goal arms, which were randomly assigned across trials (Figure 2D). This group controlled for the possibility that subjects might find the reward by attending to odor or other uncontrolled variables.

To control for possible preferences for a specific place and/or for a particular turn, half of the animals in each experimental group were trained to choose a specific turn or place, and the other
A. Allocentric Procedure

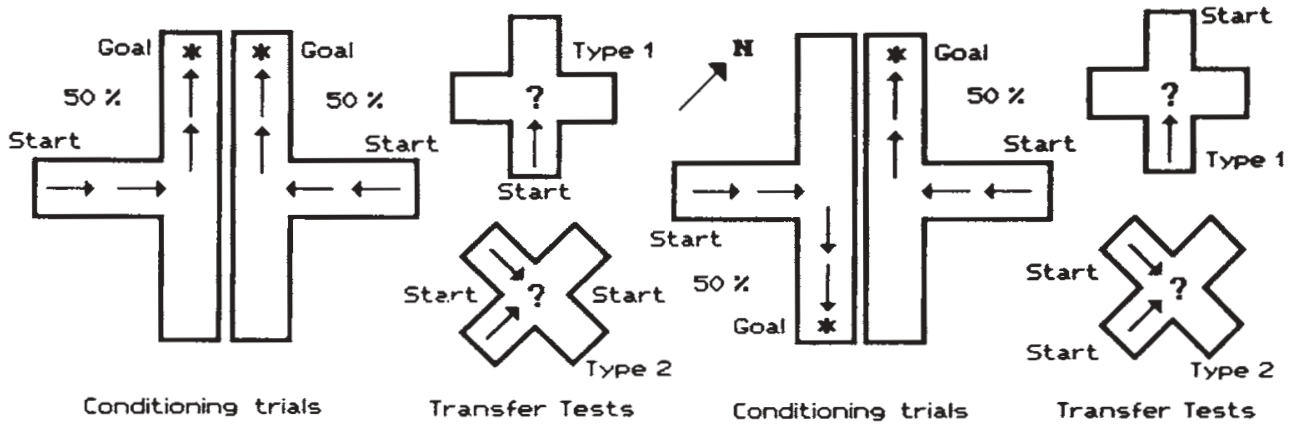

\section{Ego-Allocentric Procedure}

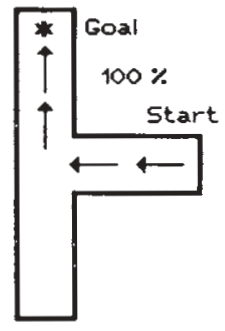

Conditioning trials

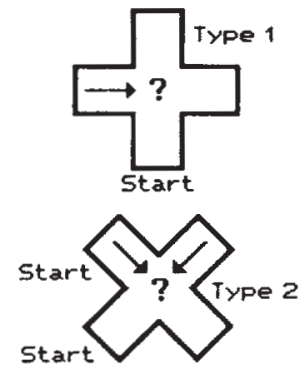

Transfer Tests
D. Control

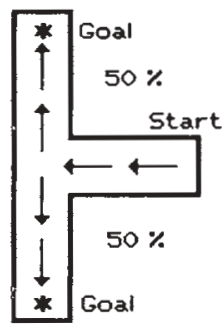

Conditioning trials
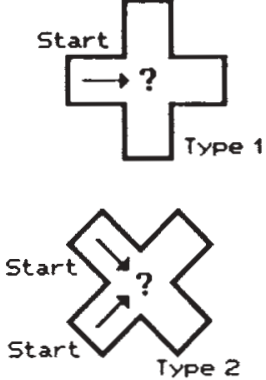

Transfer Tests

Figure 2. Schematic diagrams of the four conditioning procedures, showing also Type 1 and 2 transfer tests for each group. Trajectory examples are given for one half of each experimental group. For the other half, the procedure was identical, except that the goal arm was the opposite for allocentric and ego-allocentric groups and the turn conditioned was the opposite for the egocentric group. In the conditioning trials, arrows mark the most appropriate trajectories from the start to the goal (asterisks) for each group. The numbers represent the percentages of trials on which a specific combination of startbox and goal arm were used. In Type 1 and 2 transfer tests, the arrows denote the start arms used. 
half were trained to choose the opposite. For example, 4 animals in the egocentric group were always rewarded in the arm situated to the left of the start arm, and the other 4 were rewarded in the arm located to the right.

The fish in all groups were individually trained in daily sessions of five consecutive trials. To begin a trial, the fish was gently placed in the startbox with a small net and confined there for $20 \mathrm{sec}$. Then the guillotine door was raised and lowered after the fish left the startbox, which allowed it to swim freely in the accessible arms of the maze. A choice was recorded when the tail of the fish crossed the entrance of one arm. The fishes were left in the maze until they obtained reward (correction procedure) or until 2 min were spent. Even though the trial was considered successful only when the initial choice was correct, all errors were recorded as well as the time to get food. Thus, it was possible for an animal to make several errors on a given trial. All errors after the first one were scored as error perseverations. Once the trial was finished, the animal was removed from the arm and placed into a container during a 2-min intertrial interval. For each new trial, the fish was re-placed into the startbox, and, in a random sequence, the experimenter rebaited the corresponding food holder and simulated the rebaiting of the other food holder to control for unintentional cuing.

The training phase lasted 54 sessions (270 trials). An acquisition criterion of 13 correct trials out of 15 (a mean of $86.67 \%$ correct over three consecutive sessions) was established. When the experimental groups reached criterion, the next session was considered to be the beginning of overtraining, during which the transfer- and probe-trial tests described below were also run.

Transfer tests. The transfer trials were aimed at elucidating whether the animals of the different groups solved their respective tasks on the basis of turn or place responses. All four arms of the maze were opened during these transfer trials, and all food holders were removed (i.e., the animals were not reinforced). The transfer trials were of two types. For Type 1 trials, the maze remained in its usual position in the room, but the animals were released from a startbox located in a novel place. For Type 2 trials, the maze was displaced in the room in such a way that the end of one arm was located in the same place in the room where the fish was rewarded during training trials, but the startbox was situated in a place in the room different from where it was situated in training trials (cf. Fig- ures 1 and 2). The locations of the startbox for these trials are shown in Figures 2A-2D. For both types of trials, the first choice was recorded and then the fish was removed from the maze. Ten transfer trials ( 5 of each type) were randomly intercalated among the trials of the overtraining period-but never more than 1 per session, with a minimum of 5 conditioning trials between them. According to the arm chosen, the choice was designated as egocentric, allocentric, or other. Egocentric choices were scored when the animal made the same turn that was rewarded during the training trials, irrespective of the start place within the room. Allocentric choices were scored when the animal reached the same place in the room at which it had been rewarded in the training trials, irrespective of the start place and turn direction. All choices that could not be classified into the two latter categories were considered other.

Probe tests. To test the relevance of the distal visual cues in the solution of the different tasks, two types of probe trials were also run. For Type 1 probes, one of the most salient cues in the experimental room (e.g., the poster) was removed or hidden with brown curtains on each trial. For Type 2 probes, a brown curtain was placed around the entire maze to exclude all the cues. Ten of these probe trials ( 5 of Type 1 and 5 of Type 2) were randomly intercalated during overtraining trials, with a minimum of 5 conditioning trials between them. The location of the maze and the procedures used for each group for the probe tests were the same as those during the training trials, except that the food holders were removed from the maze (i.e., animals were not reinforced). The first arm choice was recorded and considered as correct if it coincided with those reinforced during training.

Reversal. Following Session 54, the location of food was reversed. For ego-allocentric and allocentric groups, the reversal consisted of a $180^{\circ}$ shift in the rewarded arm location. For the egocentric group, the turn opposite to that previously conditioned was rewarded. For the control group, the baited goal arm was randomly assigned during this phase. All other spatial characteristics of the experimental situation remained unchanged, as did all other procedural details used during the training period. The reversal phase lasted for 15 sessions ( 75 trials).

Data analysis. To evaluate the different characteristics of the spatial learning processes involved in the solution of each task,

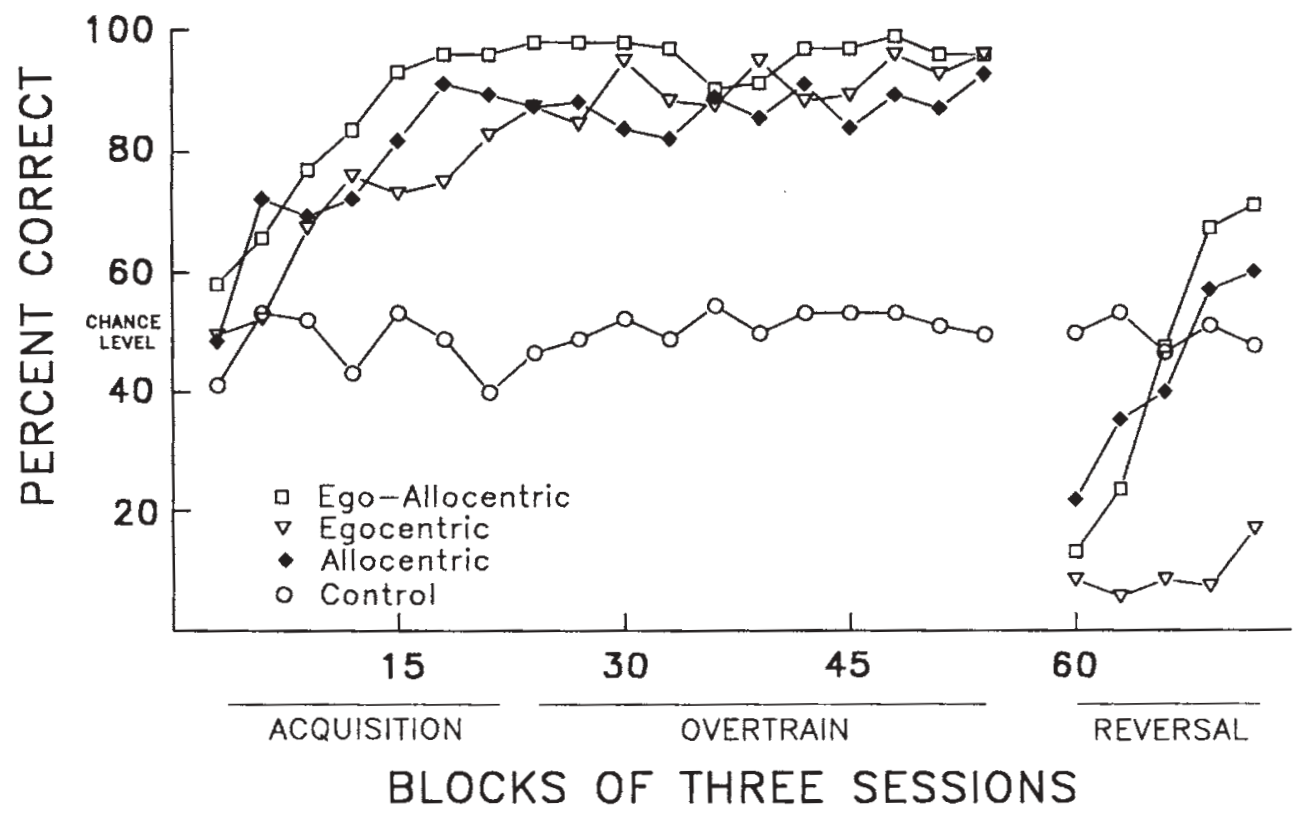

Figure 3. Percentage of correct choices for each group during the experiment. Symbols represent means of three sessions. 
several measures of performance were obtained: percentage of initial correct choices, days to reach criterion, errors to reach criterion, time spent to get food, and error perseverations. The group means for each measure were compared in nonparametric tests. The following statistical tests were used to analyze between- and within-group differences: Kruskal-Wallis one-way analysis of variance (ANOVA), Mann-Whitney $U$, Friedman two-way ANOVA, chi-square, and Wilcoxon matched-pairs signed-ranks test.

\section{RESULTS}

\section{Acquisition, Overtraining, and Reversal}

The accuracy of each group during acquisition, overtraining, and the later reversal is shown in Figure 3. There were no significant differences between the counterbalanced conditions within each group in any phase of the experiment (Mann-Whitney $U \mathrm{~s}>8$, all $p \mathrm{~s}>.19$ ), so these are collapsed in the group averages.

The percentage of correct choices remained at or near chance level in the first few sessions of the experiment, and no significant between-group differences [KruskalWallis $\chi^{2}(3)=4.23, p>.23$ ] were found for the first three sessions. On subsequent sessions, the control group remained at chance, whereas the experimental groups progressively increased their accuracy and showed significant differences relative to the control group from Sessions 4 to 20 (Mann-Whitney: allo vs. control $U=$ $1.0, p<.01$; ego vs. control $U=3.0, p<.01$; ego-allo vs. control $U=1.0, p<.01$; allo = allocentric group, ego $=$ egocentric group, and ego-allo = ego-allocentric group). In addition, there were significant differences

A.

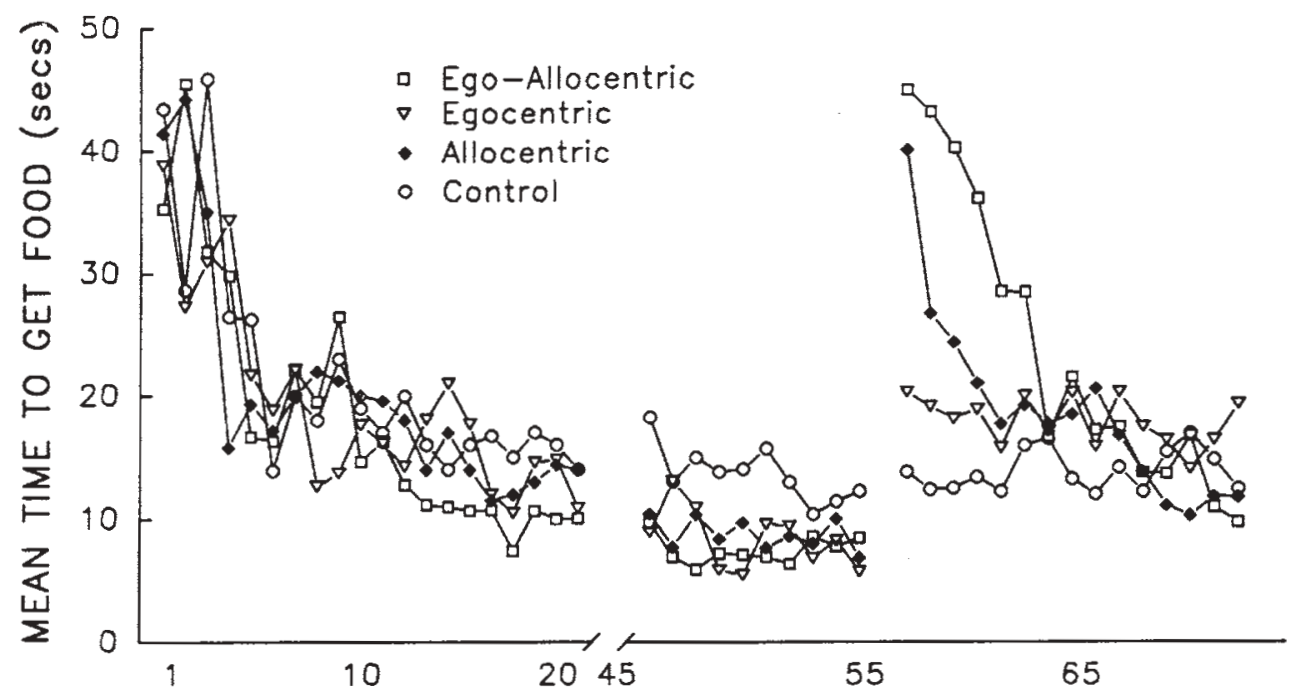

B.

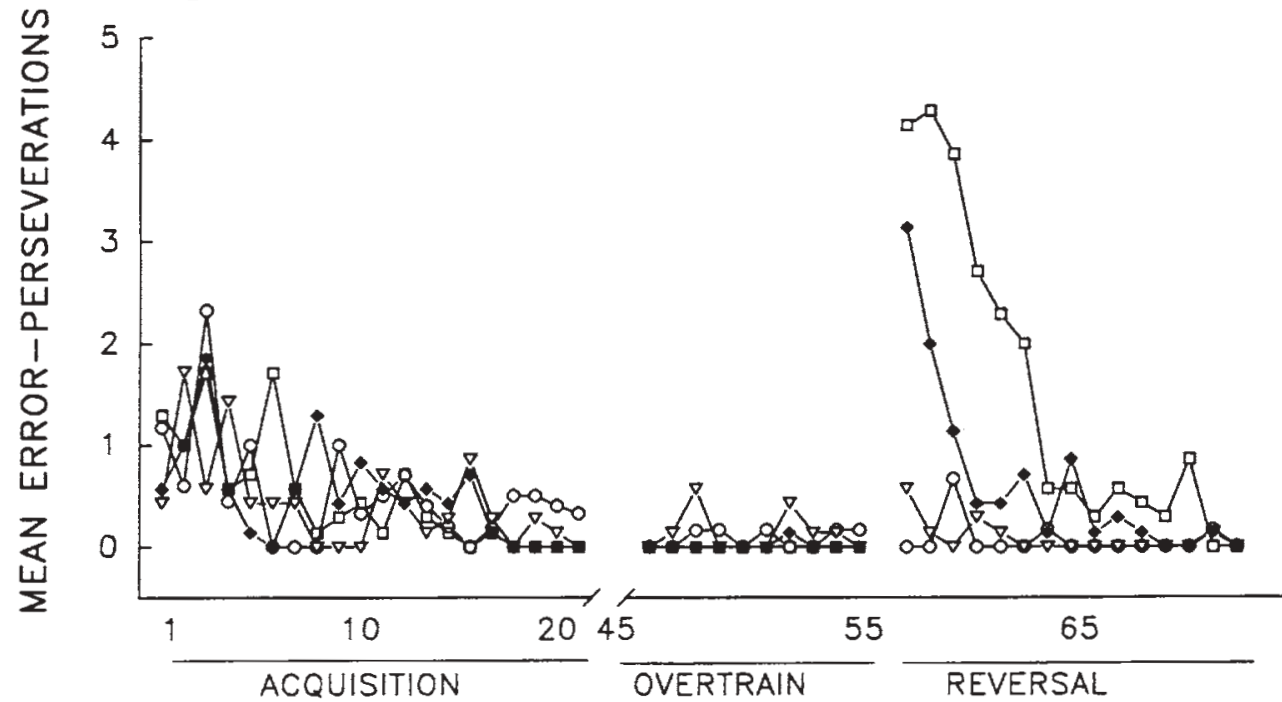

Figure 4. Mean time to get food (A) and mean number of error perseverations (B) for each group during the experiment. 
between experimental groups. Animals trained in the egocentric procedure required more sessions of training $($ ego $=20 ;$ allo $=16$; ego-allo $=14)$ and made significantly more errors $($ ego $=33.57 \pm 8.8$; allo $=23.43 \pm 6.9$; ego-allo $=19.6 \pm 4.4)$ to reach criterion relative to the other experimental groups (Mann-Whitney: ego vs. allo $U=12.8, p<.05$; ego vs. ego-allo $U=8.0, p<.05)$. The difference between the ego-allocentric group and the allocentric group was not significant.

During the overtraining phase, the experimental groups maintained a steady and high level of accuracy in the solution of their tasks (allo, 87.8\% $\pm 7 \%$; ego, 91\% $\pm 6.1 \%$; ego-allo, 96\% $2.2 \%$ ). No significant difference was found between groups during this phase [Kruskal-Wallis $\left.\chi^{2}(2)=2.62, p>.27\right]$. These three groups continued to show significantly better performance than did controls (Mann-Whitney $U_{\mathrm{s}}<6.0$, all $p \mathrm{~s}<.01$ ), which remained close to chance level $(51.03 \% \pm 4.5 \%)$. The control data show that chemosensory cues, direct visual location of the food, or other uncontrolled variables were not responsible for the accuracy level observed in the experimental groups.
Following the overtraining sessions and the transfer/ probe trials (discussed below), the location of food was reversed in each group. This change produced a dramatic decrease in the level of correct choices for all experimental groups, whereas performance in the control group was (of course) unmodified. Over the course of reversal training, differences among the experimental groups emerged as well (see Figure 3).

At the beginning of the reversal phase, accuracy in the experimental groups dropped from a level of $83 \%-$ $100 \%$ during overtraining to $4 \%-21 \%$. Of course, these differences were significant for the allocentric, egocentric, and ego-allocentric groups (Wilcoxon $Z s>2.36$, all $p \mathrm{~s}<.05)$. By contrast, the overtraining versus reversal difference in the control group was not significant (Wilcoxon $Z=1.06, p>.68$ ). It was also noteworthy that the percentage of correct choices in each experimental group was significantly lower than that for the control group (Mann-Whitney $U_{\mathrm{s}}<3.2$, all $p \mathrm{~s}<.01$ ).

The level of accuracy for the allocentric and egoallocentric groups increased quickly during reversal, whereas it remained very low for the egocentric group
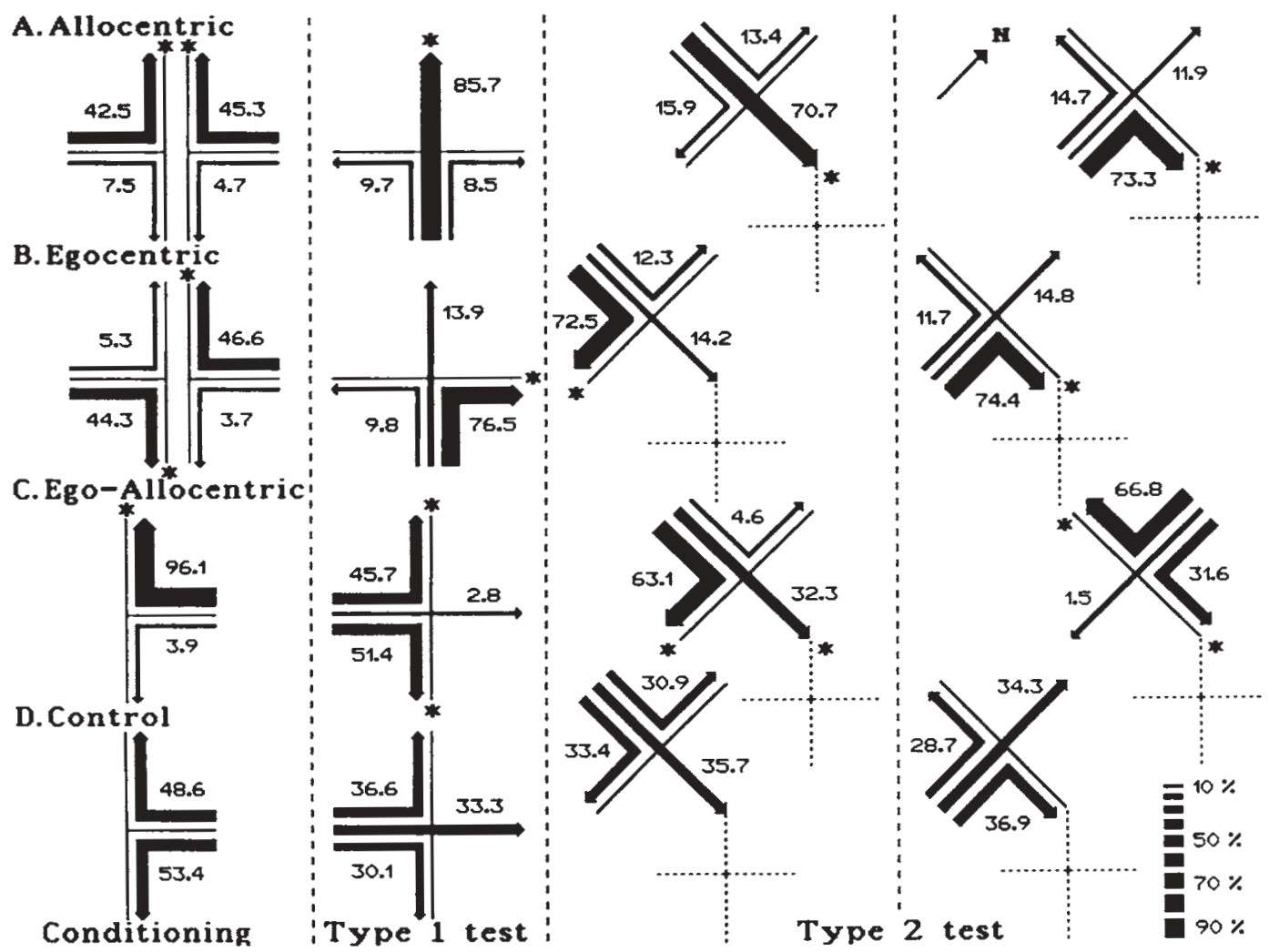

Figure 5. Schematic representation of the trajectories chosen during the transfer tests for each group. Conditioningtrial performance during overtraining is shown in the left part of the figure. Arrows indicate trajectories chosen from the starting place; their relative thicknesses and numbers denote the percentages of times that the choice was made. Solid lines represent the position of the maze for conditioning and transfer trials; dashed lines denote the normal position of the maze before its displacement for Type 2 tests. The asterisks mark the theoretical goal if the animals of allocentric and egocentric groups were using a place or orientation response, respectively, and if both responses were present for the egoallocentric group. 

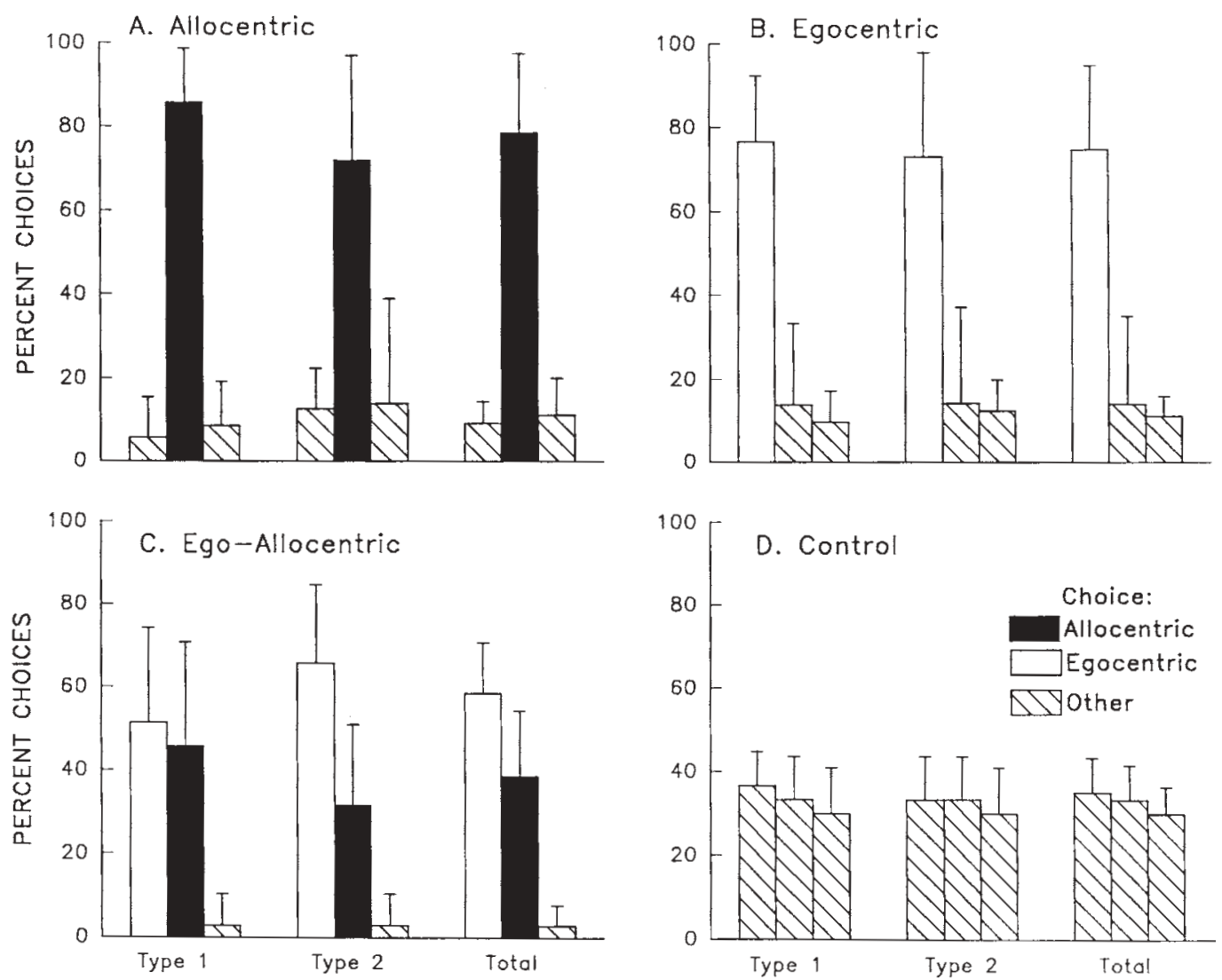

Figure 6. Mean percentage of choices in Type 1 and Type 2 transfer tests and their average (total) for each group. Each bar corresponds to one of three types of choices as described in the text.

throughout this period (Figure 3). After 1 week, statistically significant differences were found between the allocentric and ego-allocentric groups versus the egocentric group (Mann-Whitney $U_{\mathrm{s}}=2.2$ and 3.1, respectively, $p \mathrm{~s}<.01)$. At the end of the reversal phase, the former two experimental groups performed significantly more accurately than the control group (Mann-Whitney $U_{\mathrm{s}}=$ 9.07 and 6.9 , respectively, $p \mathrm{~s}<.05$ and .01$)$. Interestingly, the egocentric group did not show any significant increase in accuracy relative to the level shown at the beginning of this period (Wilcoxon $Z=.89, p>.30$ ).

During acquistion, all groups showed a decrease in the time to get food relative to initial levels, and significant differences were established between this phase and subsequent overtraining (Wilcoxon $Z \mathrm{~s}>2.03$, all $p \mathrm{~s}<.05$; see Figure 4A). At the onset of reversal, a large increase in the time to get food was observed in allocentric and ego-allocentric groups in comparison with their performance in the previous phase (both Wilcoxon $Z \mathrm{~s}=2.36, p<.05$ ), with the egocentric group (MannWhitney $U \mathrm{~s}=11.3$ and 5.62, respectively, $p \mathrm{~s}<.05$ and .01 ) and with the control group (Mann-Whitney $U_{\mathrm{s}}=$ 12.01 and 9.54 , respectively, $p$ s $<.05$ ). The time to get food in the allocentric and ego-allocentric groups decreased quickly, however, eliminating the significant differences from prereversal levels (Wilcoxon $Z \mathrm{~s}=1.12$ and 1.41, respectively, $p \mathrm{~s}>.17)$ and from the control group (both Mann-Whitney $U \mathrm{~s}>24.4, p>.25$ ).

Error perseverations diminished during acquisition and overtraining in every group, reaching values close to zero (see Figure 4B). At the onset of the reversal, the allocentric and ego-allocentric groups greatly increased their error perseverations relative to those in the overtraining phase (both Wilcoxon $Z \mathrm{~s}=2.20, p<.05$ ) and relative to those in control and egocentric groups (MannWhitney $\left.U_{\mathrm{s}}<11.7, p \mathrm{~s}<.05\right)$. However, these error perseverations rapidly decreased to their prereversal levels, eventually dropping to levels similar to those in the egocentric and control groups [Kruskal-Wallis $\chi^{2}(3)=4.17$, $p>$.24]. Error perseveration levels in the egocentric and control groups were similar during overtraining and reversal periods (Wilcoxon $Z \mathrm{~s}=.16$ and $.13, p \mathrm{~s}>.8$ ).

\section{Transfer Trials}

Figures 5 and 6 summarize the data from the two types of transfer-test trials. In the control group, the choice frequency of the three arms was close to chance on both Type 1 and Type 2 trials [both $\chi^{2} \mathrm{~s}(2)=.35, p>.83$ ], indicating a lack of a predominant preference for an arm or place (see Figures 5D and 6D). By contrast, in the egocentric and allocentric groups, the choice frequency of the three arms was significantly different from that 


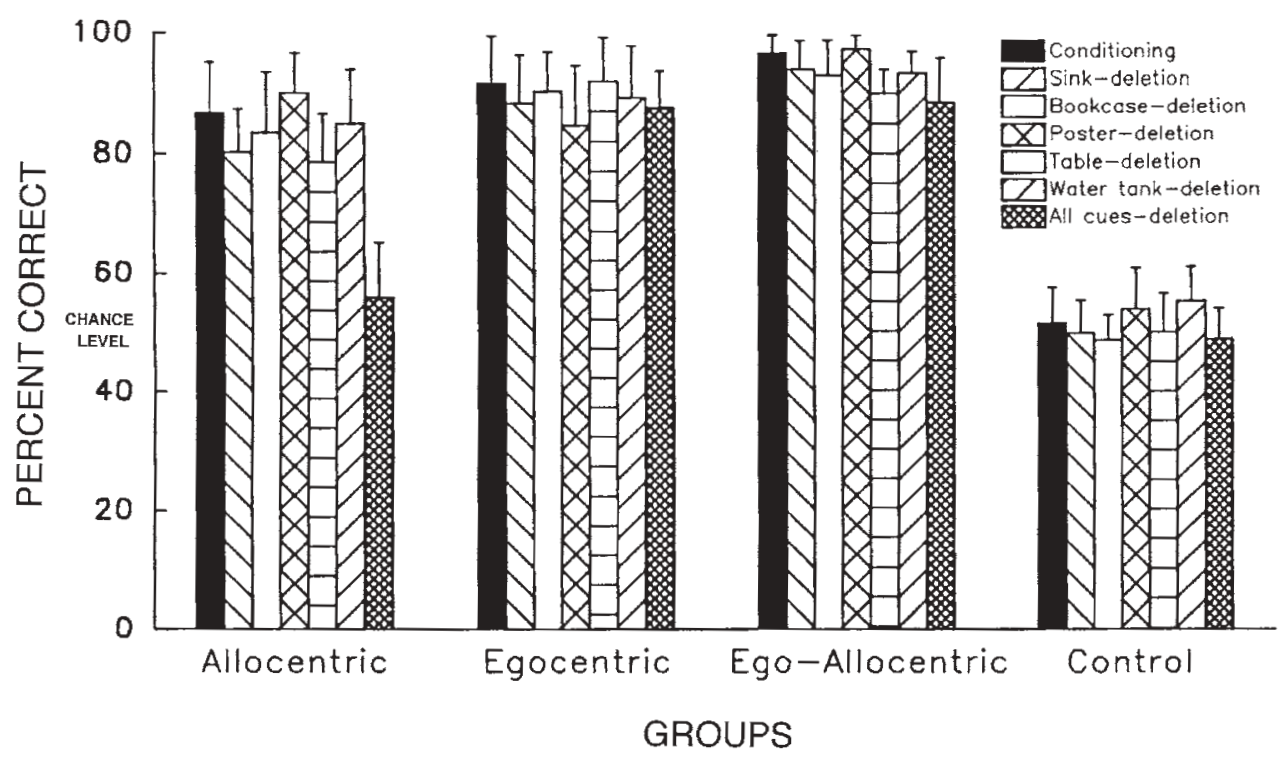

Figure 7. Percentage of correct choices in probe tests for each group when one salient cue was hidden or removed from the room, and when all distal cues were removed by curtains surrounding the maze. The percentage of correct choices during conditioning is also shown for comparison.

expected by chance on both types of trials [ego, $\chi^{2} \mathrm{~s}(2)=$ 31.4 and $24.35, p$ s $<.001$, for Type 1 and Type 2, respectively; allo, $\chi^{2} \mathrm{~s}(2)=48.05$ and $24.35, p \mathrm{~s}<.001$, for Type 1 and Type 2, respectively]. For the egocentric group, the arm most often chosen was that coinciding with its learned turn independently of the location of the startbox in the experimental room (see Figures 5B and 6B). In the Type 1 transfer test, the fish in this group showed a significant preference for the arm corresponding with an egocentric response $\left[76.5 \% \pm 15.7 \% ; \chi^{2}(1)=10, p<\right.$ $.01]$. In the Type 2 transfer test, in which the maze was displaced in the room and the fish started from an unfamiliar place, the fish also showed a strong and significant preference for the arm corresponding with the learned turn $\left[73.45 \% \pm 23 \% ; \chi^{2}(1)=6.4, p<.05\right]$. By contrast, animals in the allocentric group consistently chose the arm whose extreme was at the same place of the room where the fish was rewarded during conditioning trials $(85.7 \% \pm 13 \%$ and $72 \% \pm 19.6 \%$ for Type 1 and Type 2 tests, respectively; see Figures 5A and 6A). This tendency was significant in both tests $\left[\chi^{2}(1)=19.6\right.$ for Type $1, p<.001 ; \chi^{2}(1)=6.4$ for Type $\left.2, p<.05\right]$.

During the transfer trials for the ego-allocentric group, the positions of the maze and the startbox in the room were changed so that a turn response was incompatible with a place response. The choice frequency in this group was also significantly different from that expected by chance on both types of trials $\left[\chi^{2} \mathrm{~s}(2)=14.6\right.$ and 23.45 for Type 1 and Type 2, respectively, $p$ s $<.001]$. In the Type 1 test (see Figures 5C and 6C), fish chose the arm corresponding with the same turn they had made on rewarded training trials (an egocentric choice) on $51.4 \% \pm 22.6 \%$ of these trials, and chose the arm situated in the same place as that rewarded during training (allocentric choice) on $45.7 \% \pm 23.1 \%$ of the trials. In the Type 2 test, the egocentric $(65.71 \% \pm 19.02 \%)$ and allocentric $(31.95 \% \pm 18.1 \%)$ choices again occurred more often than the remaining choices. No significant difference was found between egocentric versus allocentric choices in the Type 1 test $\left[\chi^{2}(1)=.105, p>.74\right]$, whereas there were more egocentric than allocentric choices in the Type 2 test $\left[\chi^{2}(1)=4.33, p<.05\right]$.

\section{Probe Trials}

The probe-trial data are shown in Figure 7. When each of the salient cues in the room was individually removed or hidden, performance was not significantly affected in any group [Friedman $\chi^{2} \mathrm{~s}(5)=4.5,2.29,3.11$, and 6.73, for allocentric, egocentric, ego-allocentric, and control groups, respectively, all $p \mathrm{~s}>.35]$. However, when all cues were deleted by surrounding the maze with thick curtains, the mean percentage of correct responses in the allocentric group significantly differed from performance during conditioning, decreasing to close to chance level (Wilcoxon $Z=2.31, p<.05$ ), whereas performance in the other three groups remained unchanged (Wilcoxon $Z \mathrm{~s}<1.07$, all $p \mathrm{~s}>.30$ ).

\section{DISCUSSION}

The present experiment focused on whether goldfish could learn to solve spatial tasks by employing allocentric frames of reference and, more generally, inquired whether parallel spatial learning and memory systems with the properties described by the cognitive mapping theory of O'Keefe and Nadel (1978) might be present in 
fishes. These issues will be first discussed in view of the transfer results. Afterward, other questions such as the relative difficulty of each task, the use of distal visual cues, and the effect of reversal will be addressed.

\section{Use of Allocentric and Egocentric Strategies As Revealed by Transfer Tests}

The most noteworthy results of this experiment come from the accurate performance of fishes trained in the allocentric procedure and their behavior in the transfer trials. These fishes were able to reach the goal even when released from novel start arms (transfer test, Type 1) and from unvisited locations of the room (transfer test, Type 2). These results suggest that animals in this group made place responses by using allocentric frames of reference. Their ability to spontaneously choose the appropriate trajectory to the goal place from novel start locations and to use new routes without a history of previous training (see trajectories in Figure 5A) indicates the capacity to discriminate and represent spatial relationships in the environment independently of a body-centered reference system.

By contrast, other strategies such as the use of guidance (i.e., approaching or avoiding a particular cue; see O'Keefe \& Nadel, 1978) cannot explain the results obtained for the allocentric group, particularly those from the transfer test, Type 2. If these animals used a guidance strategy, they should have moved toward a single distal cue that served as a reference object. Instead, in the Type 2 transfer test, they navigated preferentially in a reverse direction to the place where they were reinforced during training. Of course, the possibility of a guidance strategy was minimized procedurally by avoiding the use of salient cues associated with the extremes of the goal arms. The success of this procedure was illustrated by the results of the probe trials in which particular cues were removed. On probe trials, the fish still chose correctly in the absence of any single salient distal cue.

On the other hand, several species of fish may use different compass senses to orient themselves (Quinn, 1980; Quinn \& Brannon, 1982; Quinn, Merrill, \& Brannon, 1981; Walker, 1984). Even so, the transfer data obtained here indicate that the fishes were not solving the tasks by simply employing a "direction sense" (e.g., a geomagnetic sense), because they navigated toward the goal from different directions. These results are not surprising, given that deliberate attempts to condition goldfish to respond to a magnetic field have been unsuccessful (Walker \& Bitterman, 1986).

Finally, the accurate performance of the allocentric group during training could have been realized through the use of two conditional response strategies: (1) when beginning from one start arm, to turn to the right, and (2) when beginning from the other, to turn to the left (Blodgett \& McCutchan, 1947; Ingle \& Sahagian, 1973; Thinus-Blanc \& Ingle, 1985). Again, however, the transfer tests rule out this possibility as being necessary to performance (see Olton, 1979), because the fishes went to the goal irrespective of their starting point.
Therefore, it seems that the allocentric group indeed used allocentric frames of reference to solve their task. In conjunction with previous data showing, for example, the ability of goldfish to maintain a constant environmental direction independently of the entrance point (Ingle $\&$ Sahagian, 1973) and their use of landmarks as indirect reference points (Warburton, 1990), the data from the present study indicate a cognitive mapping system like that proposed by O 'Keefe and Nadel, for a lower vertebrate group (cf. Aronson, 1951, 1971; Reese, 1989; Teyke, 1989). This cognitive mechanism could be relevant for fish for traveling in their natural habitat, especially for species that are relatively site attached, such as goldfish and common carp (Reynolds, 1983), or for those that perform repeated migrations to different areas of their habitat (Dodson, 1988; Reese, 1989). Certainly, such a cognitive system would permit flexible and adaptive spatial behaviors, such as those observed here.

However, it should be mentioned that there is a serious controversy about the nature of spatial representation supporting place responses. In addition to the hypothesis of allocentric spatial representations (Biegler \& Morris, 1993; Nadel, 1991; Worden, 1992), several authors propose that place responses might be explained by mechanisms involving egocentrically referenced representations of landmarks. For example, it has been suggested that animals can be guided by stored information about the retinal size of each of several landmarks in the room, as viewed from the goal (Leonard \& McNaughton, 1990; McNaughton, 1987). Another interesting hypothesis (Collet, Cartwright, \& Smith, 1986) suggests a goalcentered spatial memory, consisting of a set of vectors describing the distance and direction from the goal to each landmark.

The performance of fishes in the egocentric group, like that of those in the allocentric group, also showed a high level of accuracy despite a very different strategy. As is shown in Figure 5B, animals in this group mainly choose the arm corresponding to the $90^{\circ}$ turn made in training independently of the start point and of maze location in the room. Thus, it seems that the choices of these animals were purely egocentric, and that environmental information was not taken into account for the arm selection. Following the terminology of O'Keefe and Nadel (1978), these animals employed a taxon system and, more specifically, an orientation strategy.

Thus, the data from the two groups described above show that goldfish can use either egocentric or allocentric strategies to solve spatial tasks. Moreover, the results of the ego-allocentric group indicate that fish can also use both strategies simultaneously. During transfer, the animals in the latter group chose the two arms corresponding with either egocentric or allocentric choices. This pattern was not the result of random processes, because the third arm corresponding to other strategies was scarcely chosen. The use of both egocentric and allocentric strategies could explain the tendency of this group to perform more accurately and steadily than the remaining groups. The cooperative use of different spa- 
tial strategies has been suggested for fishes (Roitblat, Tham, \& Golub, 1982) and mammals (Schenk \& Morris, 1985; Whishaw, 1989; Whishaw \& Mittleman, 1986) and has been proposed for animals in their natural environment (Able, 1991; Reese, 1989). Usually, nature does not provide conflicting spatial information, so one might expect that the use of multiple behavioral systems would increase navigational efficiency in an environment with redundant cues (Able, 1991). Finally, the combined use of egocentric and allocentric strategies agrees with the proposal that "cognitive and noncognitive systems are not mutually exclusive, since they can act in concert" (O'Keefe \& Nadel, 1978, p. 520).

As mentioned before, the experimental groups could use egocentric and/or allocentric strategies to solve spatial tasks. The differences in number of errors and days to criterion in acquisition indicate that the task requiring an egocentric solution was more difficult to learn. Similar results have been described in mammals. For example, rodents trained to turn in one direction in a maze in which the environmental and intramaze cues were irrelevant were slower to learn the task than those trained in a task in which a particular place was rewarded (Hill \& Thune, 1952; Scharlock, 1955; Tolman, Ritchie, \& Kalish, 1946). These results have been explained by the disturbing effect of extramaze cues in the response group (Blodgett \& McCutchan, 1947) or by assuming that rats use a place strategy during initial learning and only shift to a response strategy with continued training (Hicks, 1964; Means \& Douglas, 1970; O’Keefe \& Nadel, 1978, 1979; N. S. Sutherland \& Mackintosh, 1971). This account could also explain why the ego-allocentric and allocentric groups learned most rapidly in the present study.

\section{Relevance of Visual Cues to Solving Allocentric Procedure}

Probe trials in which the most salient visual cues were removed or hidden also provided interesting evidence about the characteristics of the spatial strategies. No effects were observed when any single cue was taken away, but when all cues were removed, the performance of the allocentric group deteriorated, becoming as poor as that of the control group. These results suggest that the behavior of the allocentric group was controlled by a combination of many environmental cues, in agreement with the cognitive mapping system described by O'Keefe and Nadel's (1978) theory. According to this theory, such a system defines a place by its spatial relationships to a number of landmarks, none of them being essential by itself. Similar evidence consistent with this cognitive mapping view has been shown by altering the extramaze stimuli (Mazmanian \& Roberts, 1983; Morris, 1981; O'Keefe \& Conway, 1978; Suzuki et al., 1980).

On the other hand, choices of the egocentric group were not affected by removing any one cue or all of them, suggesting that the behavior of these animals was scarcely controlled by environmental stimuli. Rather, the animals apparently performed on the basis of behavioral stereotypes. Such stereotypes were probably acquired with extensive training, as reported previously for mammals (Hicks, 1964; Mackintosh, 1965; Restle, 1957), and could correspond to a pure orientation strategy in which the animals solve the task by performing a particular turn within a body-centered reference system (O’Keefe \& Nadel, 1978).

For the ego-allocentric group, the probe-trial data again suggested the use of both a cognitive mapping and a taxon strategy. Animals in this group continued to choose correctly, despite the absence of salient distal cues. Apparently, with visual cues absent, these animals relied on the taxon system. Others have reported that for subjects using simultaneous strategies to solve a spatial task, if one strategy is blocked, the animal still performs correctly on the basis of the remaining one (O'Keefe \& Nadel, 1978; Quinn, 1980; Quinn \& Brannon, 1982; Schenk \& Morris, 1985; R. J. Sutherland \& Rudy, 1989; Whishaw, 1989).

\section{Response to Reversal for Each Experimental Group}

The reacquisition data following reversal revealed other between-group differences related to the spatial strategy in each group. For example, it is consistent with the test data suggesting that the egocentric group used an orientation strategy that fishes in this group persistently chose the arm coinciding with the same turn after which they were previously rewarded.

The lack of flexibility shown by fish using egocentric strategies notably contrasts with the capacity of the allocentric groups to quickly modify their behavior during reversal. Thus, whereas the fishes in the egocentric group only reached the new goal after visiting the unrewarded arm (formerly the rewarded arm), the fishes in the allocentric and ego-allocentric groups quickly learned to move toward the new goal by using the shortest trajectory. The reversal performances of the latter two groups reveal a capacity for rapidly detecting environmental changes (Poucet, Chapuis, Durup, \& Thinus-Blanc, 1986; Thinus-Blanc et al., 1987; Welker \& Welker, 1958). Warburton (1990) reported similar reversal learning differences in goldfish trained in an arena tank to find reward in either a directly or an indirectly cued location.

\section{CONCLUDING REMARKS}

The present findings indicate that goldfish are able to establish novel routes toward a goal even from unfamiliar start points, to use distal visual cues as a whole to navigate accurately, and to reorganize their spatial strategies during reversal. These data suggest that goldfish build complex spatial cognitive representations of their environment, which permit flexibility in their spatial behavior. The data also suggest that an allocentric cognitive system works in parallel with, and can cooperate with, egocentric systems in such a way that goldfish can use the most profitable strategy in each situation. These findings are in agreement with the current notion of multiple parallel learning systems and support the generalization of the spatial learning and memory theory of 
O'Keefe and Nadel to apply to a lower vertebrate group. The possible presence of a cognitive mapping system in fishes encourages the study of its comparative peculiarities and neural substratum and encourages the notion that complex spatial cognitive systems appeared very early in the evolutionary history of vertebrates.

\section{REFERENCES}

Able, K. P. (1991). Common themes and variations in animal orientation systems. American Zoologist, 31, 157-167.

Aronson, L. R. (1951, January 17). Orientation and jumping behavior in the gobiid fish Bathygobius soporator. American Museum Novitates (No. 1486), pp. 1-22.

Aronson, L. R. (1971). Further studies on orientation and jumping behavior in the gobiid fish Bathygobius soporator. In H. E. Adler (Ed.), Orientation: Sensory bases (Annals of the New York Academy of Sciences, Vol. 188, pp. 378-392). New York: New York Academy of Sciences.

BIEgler, R., \& Morris, R. G. M. (1993). Landmark stability is a prerequisite for spatial but not discrimination learning. Nature, $\mathbf{3 6 1}$ 631-633.

Bingman, V. P. (1990). Spatial navigation in birds. In R. Kesner, \& D. S. Olton (Eds), Neurobiology of comparative cognition (pp. $423-$ 477). Hillsdale, NJ: Erlbaum.

Bingman, V. P., Bagnoli, P., Ioalé, P., \& Casini, G. (1989). Behavioral and anatomical studies of the avian hippocampus. In V. ChanPalay \& C. Köhler (Eds), The hippocampus: New vistas (pp. 379394). New York: Alan R. Liss.

Blodgett, H. C., \& McCutchan, K. (1947). Place versus response learning in the simple T-maze. Journal of Experimental Psychology, 37, 412-422.

Collet, T. S., Cartwright, B. A., \& Smith, B. A. (1986). Landmark learning and visuo-spatial memories in gerbils. Journal of Comparative Physiology A, 158, 835-851.

Dodson, J. J. (1988). The nature and role of learning in the orientation and migratory behavior of fishes. Environmental Biology of Fishes, 23, 161-182.

Hallacher, L. E. (1984). Relocation of original territories by displaced black-and-yellow rockfish, Sebastes chrysomelas, from Carmel Bay, California. Californian Fish \& Game, 7, 158-162.

Helfman, G. S., Meyer, J. L., \& McFarland, W. N. (1982). The ontogeny of twilight migration patterns in grunts (pisces: Haemulidae). Animal Behaviour, 30, 317-326.

Helfman, G. S., \& Schultz, E. T. (1984). Social transmission of behavioral traditions in a coral reef fish. Animal Behaviour, 32, 379. 384

HicKs, L. H. (1964). Effects of overtraining on acquisition and reversal of place and response learning. Psychological Reports, 15, 459-462.

Hill, C. W., \& Thune, L. E. (1952). Place and response learning in the white rat under simplified and mutually isolated conditions. Journal of Experimental Psychology, 43, 289-297.

Ingle, D., \& Sahagian, D. (1973). Solution of a spatial constancy problem by goldfish. Physiological Psychology, 1, 83-84.

Kleerekoper, H., Matis, J., Gensler, P., \& Maynard, P. (1974). Exploratory behaviour of goldfish Carassius auratus. Animal Behaviour, 22, 124-132

LeONARD, B. J., \& McNaughton, B. L. (1990). Spatial representation in the rat: Conceptual, behavioral and neurophysiological perspectives. In R. P. Kesner \& D. S. Olton (Eds), Neurobiology of comparative cognition (pp. 363-422). Hillsdale, NJ: Erlbaum.

Mackintosh, N. J. (1965). Overtraining, transfer to proprioceptive control, and position reversal. Quarterly Journal of Experimental Psychology, 17, 26-36

MARKEVICH, A. I. (1988). Nature of territories and homing in the eastern sea-perch Sebastes taczanowski. Journal of Ichthyology, 28, 161-163.

Mazmanian, D. S., \& Roberts, W. A. (1983). Spatial memory in rats under restricted viewing conditions. Learning \& Motivation, 14, 123-139.
McNaughton, B. L. (1987). Neural associations of movements and space: Preliminary steps toward a non-cartographic theory of spatial representation and learning. Neuroscience Letters, 29, S143S144.

Means, L. W., \& Douglas, R. J. (1970). Effects of hippocampal lesions on cue utilization in spatial discrimination in rats. Journal of Comparative \& Physiological Psychology, 73, 254-260.

Morris, R. G. M. (1981). Spatial localization does not require the presence of local cues. Learning \& Motivation, 12, 239-260.

NAdEL, L. (1991). The hippocampus and space revisited. Hippocampus, 1, 221-229.

O'KeEFe, J., \& Conway, D. H. (1978). Hippocampal place units in the freely moving rat: Why they fire where they fire. Experimental Brain Research, 31, 573-590.

O'Keefe, J., \& NAdel, L. (1978). The hippocampus as a cognitive map. Oxford: Oxford University Press, Clarendon Press.

O'Keefe, J., \& Nadel, L. (1979). Précis of O'Keefe \& Nadel's The hippocampus as a cognitive map. Brain \& Behavioral Sciences, 2, 487-533.

Olton, D. S. (1979). Mazes, maps and memory. American Psychologist, 34, 583-596.

Pitcher, T. J., \& Magurran, A. E. (1983). Shoal size, patch profitability and information exchange in foraging goldfish. Animal Behaviour, 31, 546-555.

Poucet, B., Chapuis, N., Durup, M., \& Thinus-Blanc, C. (1986). A study of exploratory behavior as an index of spatial knowledge in hamsters. Animal Learning \& Behavior, 14, 93-100.

QuinN, T. P. (1980). Evidence for celestial and magnetic compass orientation in lake migrating sockeye salmon fry. Journal of Comparative Physiology A, 137, 243-248.

Quinn, T. P., \& BRANnON, E. L. (1982). The use of celestial and magnetic cues by orienting sockeye salmon smolts. Journal of Comparative Physiology A, 147, 547-552.

Quinn, T. P., Merrill, R. T., \& Brannon, E. L. (1981). Magnetic field detection in sockeye salmon. Journal of Experimental Zoology, 217, 137-142.

REESE, E. S. (1989). Orientation behavior of butterflyfishes (family Chaetodontidae) on coral reefs: Spatial learning of route specific landmarks and cognitive maps. Environmental Biology of Fishes 25, 79-86.

Restle, F. (1957). Discrimination of cues in mazes: A resolution of the "place-vs-response" question. Psychological Review, 64, 217 228.

REYNOLDS, L. F. (1983). Migrations patterns of five fish species in the Murray-Darling river system. Australian Journal of Marine \& Freshwater Research, 34, 857-871.

Roitblat, H. L., Tham, W., \& Golub, L. (1982). Performance of Betta splendens in a radial arm maze. Animal Learning \& Behavior, 10 108-114.

ScHARLOCK, D. P. (1955). The role of extramaze cues in place and response learning. Journal of Experimental Psychology, 50, 249-254.

SCHENK, F., \& Morris, R. G. M. (1985). Dissociation between components of spatial memory in rats after recovery from the effects of the retrohippocampal lesions. Experimental Brain Research, 58, 11-28.

Sutherland, N. S., \& Mackintosh, N. J. (1971). Mechanisms of animal discrimination learning. New York: Academic Press.

Sutherland, R. J., \& Rudy, J. W. (1989). Configural association theory: The role of the hippocampal formation in learning, memory, and amnesia. Psychobiology, 17, 129-144.

Suzuki, S., Augerinos, G., \& Black, A. H. (1980). Stimulus control of spatial behavior on the eight-arm maze in rats. Learning \& Motivation, 11, 1-18.

TEYKE, T. (1989). Learning and remembering the environment in the blind cave fish Anoptichthys jordani. Journal of Comparative Physiology A, 164, 655-662.

Thinus-Blanc, C., Bouzouba, L., Chaix, K., Chapuis, N., Durup, M., \& Poucet, B. (1987). A study of spatial parameters encoded during exploration in hamsters. Journal of Experimental Psychology: Animal Behavior Processes, 13, 418-427.

Thinus-Blanc, C., \& Ingle, D. (1985). Spatial behavior in gerbils (Meriones unguiculatus). Journal of Comparative Psychology, 99, 311-315. 
Tolman, E. C. (1948). Cognitive maps in rats and men. Psychological Review, 55, 189-208.

Tolman, E. C., Ritchie, B. F., \& Kalish, D. (1946). Studies in spatial learning: II. Place learning versus response learning. Journal of Experimental Psychology, 3, 221-229.

WALKER, M. M. (1984). Learned magnetic field discrimination in yellowfin tuna, Thunnus albacares. Journal of Comparative Physiology $A, \mathbf{1 5 5}, 673-679$.

WalKer, M. M., \& Bitterman, M. E. (1986). Attempts to train goldfish to respond to magnetic field stimuli. Naturwissenschaften, 73, 12-16.

WARBURTON, K. (1990). The use of local landmarks by foraging goldfish. Animal Behaviour, 40, 500-505.

WELKER, W. I., \& WELKER, J. (1958). Reaction of fish (Eucinostomus gula) to environmental changes. Ecology, 39, 283-288.

WHISHAW, I. Q. (1989). Dissociating performance and learning deficits on spatial navigation tasks in rats subjected to cholinergic muscarinic blockade. Brain Research Bulletin, 23, 347-358.

Whishaw, I. Q., \& Mittleman, G. (1986). Visits to starts, routes and places by rats (Rattus norvegicus) in swimming pool navigation tasks. Journal of Comparative Psychology, 100, 422-431.

Worden, R. (1992). Navigation by fragment fitting: A theory of hippocampal function. Hippocampus, 2, 165-188. 